\title{
INVARIANT MEASURES OF SYMMETRIC LÉVY PROCESSES
}

\author{
JIANGANG YING \\ (Communicated by Lawrence F. Gray)
}

\begin{abstract}
If $\pi=\left\{\pi_{t}: t>0\right\}$ is a symmetric convolution semigroup with the Lévy exponent $\phi$, then supp $\pi_{t}$ is a group determined by $\phi$, and $\pi$ has a unique Radon invariant measure if and only if $\phi$ has a unique zero at 0 .
\end{abstract}

\section{INTRODUCTION}

Let $X=\left(X_{t}, P^{x}\right)$ be a Lévy process on $R^{d}$ (i.e., a process with stationary independent increments), with transition semigroup $\left(P_{t}\right)$. Let $m$ denote Lebesgue measure on $R^{d}$. For any probability measure $\mu$ on $R^{d}$, define the characteristic function of $\mu$ as

$$
\hat{\mu}(x):=\int_{R^{d}} e^{i(x, y)} \mu(d y) .
$$

Let $\pi_{t}:=P_{t}(0, \cdot)$. Then $\pi=\left\{\pi_{t}\right\}$ is a convolution semigroup with Lévy exponent $\phi$; i.e., $\hat{\pi}_{t}(x)=e^{-t \phi(x)}$. By the Lévy-Khinchin formula,

$$
\phi(x)=i(a, x)+\frac{1}{2}(S x, x)+\int_{R^{d}}\left(1-e^{i(x, y)}+\frac{i(x, y)}{1+|y|^{2}}\right) J(d y)
$$

with $a \in R^{d}, S$ a $d \times d$ nonnegative definite matrix, and $J$ a positive measure carried by $R^{d}-\{0\}$ such that

$$
\int_{R^{d}}\left(1 \wedge|x|^{2}\right) J(d x)<\infty
$$

The measure $J$ is called the Lévy measure of $X$. The process $X$ is called symmetric if each $\pi_{t}$ is invariant under the map $x \mapsto-x$. In this case, $\phi$ has the form

$$
\phi(x)=\frac{1}{2}(S x, x)+\int_{R^{d}}(1-\cos (x, y)) J(d y)
$$

where $J$ is symmetric.

A $\sigma$-finite measure $\mu$ on $R^{d}$ is called an invariant measure for $X$ if $\mu * \pi_{t}=$ $\mu$ for all $t>0$. Denote by Inv the set of all invariant measures for $X$. Clearly

Received by the editors April 27, 1992.

1991 Mathematics Subject Classification. Primary 60J30; Secondary 60J25, 60B15.

Key words and phrases. Lévy processes, invariant measures.

Research supported in part by NSF Grant DMS 91-01675.

(C) 1993 American Mathematical Society $0002-9939 / 93 \$ 1.00+\$ .25$ per page 
$m \in$ Inv. We say that $X$ has a unique invariant measure if $\mu \in$ Inv implies that $\mu$ is a multiple of $m$ and that $X$ has a unique Radon invariant measure if the only Radon measures in Inv are multiplies of $m$.

In this paper, two results concerning symmetric convolution semigroups will be presented. In $\S 2$ we prove that the support of each measure $\pi_{t}$ is the group generated by the sum of the range of $\sqrt{S}$ and the support of the Lévy measure. In $\S 3$ we prove that $X$ has a unique Radon invariant measure if and only if its Lévy exponent has a unique zero at 0 . Finally, a few examples are given as an application and explanation of our results.

\section{SUPPORT OF CONVOLUTION SEMIGROUP}

The translation operators $\left\{\gamma_{x}: x \in R^{d}\right\}$ on $R^{d}$ are defined by $\gamma_{x}(y)=x+y$. For any function $f$ on $R^{d}$, let

$$
\operatorname{per} f=\left\{x \in R^{d}: f \circ \gamma_{x}=f\right\} \text {. }
$$

Clearly per $f$ is a closed subgroup of $R^{d}$ if $f$ is continuous. For any $A \subset R^{d}$, let $G(A)$ denote the closed subgroup generated by $A$ in $R^{d}$ and

$$
A^{\perp}=\left\{x \in R^{d}: e^{i(x, y)}=1 \forall y \in A\right\} .
$$

We list some facts here which will be used later. The first four are easy to check and the last one is [BF, Proposition 6.3].

(a) $A^{\perp}$ is a closed subgroup of $R^{d}$.

(b) $\left(A^{\perp}\right)^{\perp}=G(A)$.

(c) $G(A+B)=\overline{G(A)+G(B)}$.

(d) $G(A)=G(\bar{A})$.

(e) For any probability measure $\mu$ on $R^{d}$, per $\hat{\mu}=(\operatorname{supp} \mu)^{\perp}$, where supp $\mu$ denotes the support of $\mu$.

In the remainder of this section, we will assume $X$ is symmetric. Then $\phi$ has the representation (1.4). Let $\pi^{c}$ and $\pi^{d}$ be the convolution semigroups corresponding to the Lévy exponents $\frac{1}{2}(S x, x)$ and $\int(1-\cos (x, y)) J(d y)$, respectively. Then $\pi_{t}=\pi_{t}^{c} * \pi_{t}^{d}$ for all $t>0$ and

$$
\operatorname{supp} \pi_{t}=\overline{\operatorname{supp} \pi_{t}^{c}+\sup \pi_{t}^{d}} .
$$

The main result in this section is the following.

Theorem 1. For any $t>0$,

$$
\operatorname{supp} \pi_{t}=\overline{\sqrt{S}\left(R^{d}\right)+G(\operatorname{supp} J)}=G\left(\sqrt{S}\left(R^{d}\right)+\operatorname{supp} J\right) .
$$

Proof. Clearly supp $\pi_{t}^{c}=\sqrt{S}\left(R^{d}\right)$. By (2.3), it suffices to show that supp $\pi_{t}=$ $G(\operatorname{supp} J)$ if $S=0$. Now assume $S=0$. We will finish the proof in several steps.

1. For any $t>0, G\left(\operatorname{supp} \pi_{t}\right)=G(\operatorname{supp} J)$. In fact, for any fixed $t>0$, by facts (b) and (e), we only need to show that per $\hat{\pi}_{t}=(\operatorname{supp} J)^{\perp}$. Let zer $f$ denote the set of zeros of a function $f: R^{d} \rightarrow R$. Since

$$
\phi(x)=\int_{R^{d}}(1-\cos (x, y)) J(d y),
$$


$x \in \operatorname{zer} \phi$ if and only if $J$ is carried by $\{x\}^{\perp}=\{y: \cos (x, y)=1\}$ or equivalently $x \in(\operatorname{supp} J)^{\perp}$. Thus $\operatorname{zer} \phi=(\operatorname{supp} J)^{\perp}$. On the other hand, it is easy to see that per $\hat{\pi}_{t} \subset \operatorname{zer} \phi$. Conversely, if $x \in \operatorname{zer} \phi$, then $\operatorname{supp} J \subset\{x\}^{\perp}$. Thus $\cos (x, \cdot) J=J$ and $\sin (x, \cdot) J=0$, and it follows that

$$
\phi(x+y)=\int(1-\cos (x+y, z)) J(d z)=\phi(y) .
$$

Therefore, $x \in \operatorname{per} \phi \subset$ per $\hat{\pi}_{t}$.

2. $\operatorname{supp} \pi_{t}$ is a group and $\operatorname{supp} \pi_{t}=G(\operatorname{supp} J)$ for any $t>0$. By symmetry of $\pi_{t}$, it is clear that if $x \in \operatorname{supp} \pi_{t}$ then $-x \in \operatorname{supp} \pi_{t}$. We will prove $0 \in \operatorname{supp} \pi_{t}$ by contradiction. Suppose that $0 \notin \operatorname{supp}\left(\pi_{t}\right)$. Then there exists $\delta>0$ such that $\pi_{t}\left(B_{\delta}\right)=0$ where $B_{\delta}=\{x:|x|<\delta\}$. Let

$$
f(t)=\frac{1}{a}\left(1-\frac{|t|}{a}\right) 1_{[-a, a]}(t), \quad t \in R,
$$

and

$$
g\left(x_{1}, \ldots, x_{d}\right)=f\left(x_{1}\right) \cdots f\left(x_{d}\right) .
$$

Then $g$ is a continuous function on $R^{d}$ with compact support and

$$
\hat{g}(x)=\left(\frac{2}{a^{2}}\right)^{d} \frac{\left(1-\cos a x_{1}\right) \cdots\left(1-\cos a x_{d}\right)}{x_{1}^{2} \cdots x_{d}^{2}} \geq 0 \text {. }
$$

Now choose $a>0$ such that $[-a, a]^{d} \subset B_{\delta}$. Then

$$
0=\left\langle\pi_{t}, g\right\rangle=\frac{1}{(2 \pi)^{d / 2}}\left\langle\hat{\pi}_{t}, \hat{g}\right\rangle=\frac{1}{(2 \pi)^{d / 2}} \int_{R^{d}} e^{-t \phi(x)} \hat{g}(x) d x .
$$

But $e^{-t \phi(x)}>0$ everywhere. This implies that $\hat{g}=0$, which is a contradiction. Now assume that $J\left(R^{d}\right)<\infty$. Then

$$
\pi_{t}=e^{-t \beta} \sum_{n \geq 0} \frac{t^{n}}{n !} J^{* n}
$$

where $\beta=J\left(R^{d}\right)$ and $J^{* n}$ denotes the $n$-fold convolution of $J$. Thus, immediately, we have

$$
\operatorname{supp} \pi_{t}=\overline{\bigcup_{n \geq 0} \operatorname{supp} J^{* n}}
$$

By the result in step 1,

$$
G(\operatorname{supp} J)=G\left(\operatorname{supp} \pi_{t}\right) \supset \operatorname{supp} \pi_{t}=\overline{\bigcup_{n \geq 0} \operatorname{supp} J^{* n}} .
$$

It is easy to check that $\bigcup \operatorname{supp} J^{* n}$ is a group. Hence,

$$
\overline{\bigcup_{n>0} \operatorname{supp} J^{* n}}=G(\operatorname{supp} J)=\operatorname{supp} \pi_{t}
$$

since $U \operatorname{supp} J^{* n} \supset \operatorname{supp} J$.

In general, let $J_{n}$ be the restriction of $J$ to $B_{1 / n}^{c}$, which is finite and symmetric. Let

$$
\begin{aligned}
& \phi_{1}(x)=\int_{R^{d}}(1-\cos (x, y)) J_{n}(d y) \\
& \phi_{2}(x)=\int_{R^{d}}(1-\cos (x, y))\left(J-J_{n}\right)(d y)=\phi(x)-\phi_{1}(x) .
\end{aligned}
$$


Then there exist two convolution semigroups $\xi^{n}=\left\{\xi_{t}^{n}\right\}$ and $\mu^{n}=\left\{\mu_{t}^{n}\right\}$ with Lévy exponents $\phi_{1}$ and $\phi_{2}$, respectively. Clearly, $\xi_{t}^{n} * \mu_{t}^{n}=\pi_{t}$ and $\operatorname{supp} \xi_{t}^{n}=$ $G\left(\operatorname{supp} J_{n}\right)$ since $J_{n}$ is finite.

Now supp $J \backslash\{0\}=\bigcup_{n \geq 1}$ supp $J_{n}$ and

$$
G(\operatorname{supp} J)=G(\operatorname{supp} J \backslash\{0\})=G\left(\bigcup \operatorname{supp} J_{n}\right) \supset G\left(\operatorname{supp} J_{n}\right),
$$

or $G(\operatorname{supp} J) \supset \bigcup_{n \geq 1} G\left(\operatorname{supp} J_{n}\right)$. Since $\left\{G\left(\operatorname{supp} J_{n}\right)\right\}_{n \geq 1}$ is an increasing sequence of subgroups, $\bigcup_{n \geq 1} G\left(\operatorname{supp} J_{n}\right)$ is also a subgroup. Thus

$$
\overline{\bigcup_{n>1} G\left(\operatorname{supp} J_{n}\right)} \supset G\left(\bigcup_{n \geq 1} \operatorname{supp} J_{n}\right)=G(\operatorname{supp} J) \text {. }
$$

That is, $\overline{\bigcup_{n \geq 1} G\left(\operatorname{supp} J_{n}\right)}=G(\operatorname{supp} J)$.

Next,

$$
\operatorname{supp} \pi_{t} \supset \operatorname{supp} \xi_{t}^{n}+\operatorname{supp} \mu_{t}^{n} \supset \operatorname{supp} \xi_{t}^{n}
$$

since $0 \in \operatorname{supp} \mu_{t}^{n}$. Then

$$
\operatorname{supp} \pi_{t} \supset \overline{\bigcup_{n \geq 1} \operatorname{supp} \xi_{t}^{n}}=\overline{\bigcup_{n \geq 1} G\left(\operatorname{supp} J_{n}\right)}=G(\operatorname{supp} J)=G\left(\operatorname{supp} \pi_{t}\right) .
$$

Therefore, $\operatorname{supp} \pi_{t}=G(\operatorname{supp} J)=G\left(\operatorname{supp} \pi_{t}\right)$. This completes the proof. Q.E.D.

\section{RADON INVARIANT MEASURES}

Choquet and Deny [CD] investigated and solved the convolution equation

$$
\sigma * \mu=\mu \quad(\sigma \geq 0 \text { a given Radon measure })
$$

on a locally compact Abelian group. Here we will state their result only for $R^{d}$.

Let $G_{\sigma}=G(\operatorname{supp} \sigma)$, and let $\omega$ be the trivial extension to $R^{d}$ of Haar measure on $G_{\sigma}$. It is known that there exists a Borel set $\Gamma_{\sigma} \subset R^{d}$ consisting of exactly one representative from each equivalence class of the quotient group $R^{d} / G_{\sigma}$. Let

$$
E_{\sigma}:=\left\{x \in R^{d}: \int_{R^{d}} e^{-(x, y)} \sigma(d y)=1\right\} .
$$

Theorem (Choquet-Deny). Any Radon measure $\mu$ satisfying the equation (3.1) is of the form

$$
\mu=\int_{R^{d} \times R^{d}}\left(e^{(x, \bullet)} \omega\right) * \delta_{y} \xi(d x, d y)
$$

where $\xi$ is a positive Radon measure on $R^{d} \times R^{d}$ carried by $E_{\sigma} \times \Gamma_{\sigma}$ and $\delta_{y}$ is the Dirac measure at $y$.

Now for the convolution semigroup $\pi$, let

$$
\sigma=\int_{0}^{\infty} e^{-t} \pi_{t} d t
$$


Then $\sigma$ is a probability measure $R^{d}$ and $\mu \in$ Inv if and only if $\mu$ satisfies (3.1). Clearly

$$
\operatorname{supp} \sigma=\overline{\bigcup_{t>0} \operatorname{supp} \pi_{t}} .
$$

Thus $G_{\sigma}=G\left(\bigcup_{t>0} \operatorname{supp} \pi_{t}\right)$. By [BF, Proposition 8.27], $G_{\sigma}=(\operatorname{zer} \phi)^{\perp}$ and $\operatorname{zer} \phi=G_{\sigma}^{\perp}$. On the other hand, $x \in E_{\sigma}$ if and only if $\int_{R^{d}} e^{-(x, y)} \pi_{t}(d y)=1$ for all $t>0$. Obviously, there exists a $(-\infty,+\infty]$-valued function $\psi$ on $R^{d}$ such that

$$
\int_{R^{d}} e^{-(x, y)} \pi_{t}(d y)=e^{t \psi(x)}
$$

Hence $E_{\sigma}=\operatorname{zer} \psi$. It is easy to see that $0 \in \operatorname{zer} \phi$ and $0 \in \operatorname{zer} \psi$. The main result of this section is

Theorem 2. (a) The process $X$ has a unique Radon invariant measure if and only if zer $\phi=$ zer $\psi=\{0\}$.

(b) If $X$ is symmetric, then $X$ has a unique Radon invariant measure if and only if zer $\phi=\{0\}$.

Proof. (a) follows immediately from the theorem of Choquet and Deny since $E_{\sigma}=\operatorname{zer} \psi$, and $\Gamma_{\sigma}=\{0\}$ if and only if $\operatorname{zer} \phi=\{0\}$.

(b) By (a), it suffices to show that zer $\phi=\{0\}$ implies that zer $\psi=\{0\}$ if $X$ is symmetric. By way of contradiction, suppose that $a \neq 0$ and $a \in E_{\sigma}$. Then $-a \in E_{\sigma}$ by the symmetry of $\sigma$, and for any $b \in(-a, a):=\{t a: t \in(-1,1)\}$ there exist numbers $0<p, q<1$ with $p+q=1$ such that $b=(-a) p+a q$. Applying Hölder's inequality, we have

$$
\begin{aligned}
\int_{R^{d}} e^{-(b, x)} \sigma(d x) & =\int\left[e^{-(-a, x)}\right]^{p} \cdot\left[e^{-(a, x)}\right]^{q} \sigma(d x) \\
& \leq\left[\int e^{(a, x)} \sigma(d x)\right]^{p}\left[\int e^{-(a, x)} \sigma(d x)\right]^{q}=1
\end{aligned}
$$

Again by symmetry and Hölder's inequality, $\int e^{(b, x)} \sigma(d x) \leq 1$ and

$$
\begin{aligned}
1 & =\int \sigma(d x)=\int e^{-(b, x) / 2+(b, x) / 2} \sigma(d x) \\
& \leq\left[\int e^{-(b, x)} \sigma(d x)\right]^{1 / 2} \cdot\left[\int e^{(b, x)} \sigma(d x)\right]^{1 / 2} \leq 1 .
\end{aligned}
$$

Thus $\int e^{-(b, x)} \sigma(d x)=\int e^{(b, x)} \sigma(d x)=1$; i.e., $(-a, a) \subset E_{\sigma}$.

Now, for any $t \in(0,1)$,

$$
\begin{aligned}
1 & =\int e^{(t a, x)} \sigma(d x) \\
& =\int_{(a, x)<0}+\int_{(a, x)=0}+\int_{(a, x)>0} e^{(t a, x)} \sigma(d x) \\
& =c+\int_{(a, x)>0}\left(e^{(t a, x)}+e^{-(t a, x)}\right) \sigma(d x)
\end{aligned}
$$

where $c=\sigma(\{x:(a, x)=0\})$. Let $f(t, x)=e^{(t a, x)}+e^{-(t a, x)}$. Then

$$
\frac{d f}{d t}(t, x)=(a, x)\left[e^{(t a, x)}-e^{-(t a, x)}\right]>0
$$


for any $t \in(0,1)$ and $(a, x)>0$. But $\int_{(a, x)>0} f(t, x) \sigma(d x)=1-c$ does not depend on $t$. Thus $\sigma(\{x:(a, x)>0\})=0$; i.e., $\sigma$ is carried by the subspace $\{x:(a, x)=0\}$. This contradicts the assumption $a \in E_{\sigma}$ and completes the proof. Q.E.D.

The uniqueness results above for Radon invariant measures are not true in general. (See the example below.) But it is known that if $m$ is a reference measure for $X$, i.e., $\sigma \ll m$, then each invariant measure of $X$ is a Radon measure [BG, $\mathrm{VI}(2.3)]$. In this case, $G_{\sigma}=R^{d}$ and $\Gamma_{\sigma}=\{0\}$. Thus, the following result is a simple consequence of Theorem 2 .

Corollary. Assume $\sigma \ll m$. Then:

(a) $X$ has a unique invariant measure if and only if zer $\psi=\{0\}$.

(b) If $X$ is symmetric, then $X$ has a unique invariant measure.

Now we will construct an example such that any Radon invariant measure of $X$ is a multiple of Lebesgue measure but $X$ has an invariant measure which is not Radon. For the case in which $J$ is finite, we have (2.7), and it is easy to check that $\mu \in$ Inv if and only if $\frac{1}{\beta} \mu * J=\mu$.

Example 1. Let $J=\frac{1}{4}\left(\delta_{1}+\delta_{-1}+\delta_{\sqrt{2}}+\delta_{-\sqrt{2}}\right)$ be defined on $R$ and $\pi=\left\{\pi_{t}\right\}_{t>0}$ the corresponding symmetric convolution semigroup; i.e., $\hat{\pi}_{t}(x)=e^{-t \phi(x)}$ with

$$
\phi(x)=\int(1-\cos x y) J(d y)=\frac{1}{2}(1-\cos x)+\frac{1}{2}(1-\cos \sqrt{2} x) .
$$

Clearly $\phi$ has a unique zero. Thus the only Radon invariant measure of $\pi$ is Lebesgue measure. But let

$$
N=\{n+m \sqrt{2}: n, m \text { are integers }\} \text { and } \mu=\sum_{x \in N} \delta_{x} .
$$

Then $\mu$ is $\sigma$-finite and satisfies $\mu * J=\mu$. Therefore, $\mu$ is an invariant measure for $X$.

Example 2. Brownian motion and symmetric stable processes. Both are special types of symmetric Lévy processes, where the Lévy exponent $\phi(x)=\frac{1}{2}|x|^{2}$ for Brownian motion and $\phi(x)=|x|^{\alpha}, \alpha \in(0,2)$, for symmetric stable processes. Clearly both functions have unique zeros. By Corollary 3 , both processes have unique invariant measures.

Example 3. Brownian motion with drift on $R^{d}$. In this case,

$$
\phi(x)=i(x, \alpha)+\frac{1}{2}|x|^{2}, \quad \psi(x)=-(x, \alpha)-\frac{1}{2}|x|^{2},
$$

where $\alpha \in R^{d}$. Then $E_{\sigma}=\{0,-\alpha\}$ and $\operatorname{zer} \phi=\{0\}$. Thus invariant measures are of the form $\mu=c_{1} m+c_{2} e^{-\left(\alpha,{ }^{\circ}\right)} m$, where $c_{1}$ and $c_{2}$ are any positive constants.

\section{ACKNOWLEDGMENT}

I would like to thank Professors P. J. Fitzsimmons and R. K. Getoor for stimulating discussions and great help during the preparation of this paper. 


\section{REFERENCES}

[BF] C. Berg and G. Forst, Potential theory on locally compact abelian groups, Springer-Verlag, Berlin, Heidelberg, and New York, 1973.

[BG] R. M. Blumenthal and R. K. Getoor, Markov processes and potential theory, Academic Press, New York, 1968.

[CD] G. Choquet and J. Deny, Sur l'équation de convolution $\sigma * \mu=\mu$, C. R. Acad. Sci. Paris Sér. I Math. 250 (1960), 799-801.

Department of Mathematics, University of California at San Diego, la Jolla, CaliFORNIA 92093-0112

E-mail address: jying@math.ucsd.edu 